\title{
ULTRAFILTER LIMITS AND FINITELY ADDITIVE PROBABILITY1
}

\author{
THOMAS Q. SIBLEY
}

\begin{abstract}
Ultrafilter limits provide the natural convergence notion for finitely additive probability. The finitely additive infinitely divisible laws are closed under ultrafilter limits. The characteristic function of any convolution of finitely additive probability measures is the product of their characteristic functions.
\end{abstract}

Limits are generally sharp enough to provide results in countably additive probability. The weaker axiom of finite additivity needs the correspondingly stronger convergence of ultrafilter limits. See Bell and Slomson [2] for a readable introduction to model theory and ultrafilters. I am deeply appreciative for the many helpful comments of the referee and of my advisor, Professor Rohit Parikh.

$m$ is a finitely additive probability measure (abbreviated f.a.p.m.) iff (i) there is a Boolean subalgebra $\mathscr{B}$ of $\mathscr{P}(R)$ so that $m: \mathscr{B} \rightarrow[0,1]$, (ii) $R \in \mathscr{B}$ and $m(R)=1$, and (iii) $\forall A, C \in \mathscr{B}$ if $A \cap C=\varnothing$, then $m(A \cup C)=m(A)+m(C)$.

Given a family $\left\{f_{i}: i \in I\right\}, I \neq \varnothing$, of functions from a set $E$ into a compact Hausdorff space $T$ and an ultrafilter $U$ on $I$, the ultrafilter $\operatorname{limit} U-\lim \left(f_{i}: i \in I\right)$ is the function $f$ from $E$ into $T$ so that $f(e)=t$ iff for all open neighborhoods $H$ of $t$ in $T,\left\{i \in I: f_{i}(e) \in H\right\} \in U$. It is well known (as in [3]) that the properties of compactness and Hausdorff are necessary and sufficient for ultrafilter limits. Since $[0,1]$ is a compact Hausdorff space, the ultrafilter limit of a f.a.p.m. is easily seen to be a f.a.p.m.

Given a f.a.p.m. $m$, the characteristic function or Fourier Stieltjes transform (abbreviated ch.f.) of $m$ is the function $f$ from $R$ into the closed unit disk of the complexes so that $\forall t \in R, f(t)=\int_{R} e^{i t x} d m(x)$. The following lemma shows that ultrafilter limits fit naturally with characteristic functions.

Lemma. Let $\left\{m_{i}: i \in I\right\}$ be any nonempty set of f.a.p.m., $\left\{f_{i}: i \in I\right\}$ be their corresponding ch.f., and $U$ an ultrafilter on $I$. Then the ch.f. of $U-\lim \left(m_{i}: i \in I\right)$ is $U-\lim \left(f_{i}: i \in I\right)$.

Proof. The integral of any bounded function like $e^{i t x}$ is readily determined by approximations with simple functions. See Shorb [5]. Given $J$ finite and $m=U$ $\lim \left(m_{i}: i \in I\right)$, by finite additivity, $\left(\sum b_{j} m\left(A_{j}\right): j \in J\right)=U-\lim \left(\sum b_{j} m_{i}\left(A_{j}\right): j \in J\right)$. It readily follows that $U-\lim \left(f_{i}: i \in I\right)$ must be the ch.f. of $m$.

Received by the editors April 29, 1980 and, in revised form, April 17, 1981.

1980 Mathematics Subject Classification. Primary 03H05, 60B10; Secondary 03C20, 60E07, 60E10.

Key words and phrases. Finitely additive probability, ultrafilter limits, infinitely divisible laws, characteristic functions, ultrafilters, nonstandard analysis.

1 This paper is a revision of part of my Ph.D. dissertation [6] at Boston University. 
Much of the usefulness of ch.f. stems from Theorem 1 on convolutions, which correspond to the sums of independent random variables. Product measures, and so convolutions, are uniquely defined in countably additive probability. However, with finite additivity, convolutions are no longer unique. See Appling [1] for the definition of product measures for f.a.p.m. A f.a.p.m. $r$ is a convolution of the f.a.p.m. $m$ and $p$ iff there is an extension $s$ of the product measure $m \times p$ to all subsets of $R \times R$ so that $\forall A \in \mathscr{B}, r(A)=s\{(x, y): x+y \in A\}$. We will write $r=m * p$. Similarly, a $k$-fold convolution of $m, r=m^{* k}$, is obtained from the extension of the product measure $m \times \cdots \times m$.

THEOREM 1. The ch.f. of any convolution of f.a.p.m. is the product of their ch.f.

Proof. Fubini's Theorem as extended by Appling [1] provides the key to this proof. Let $f, g$, and $h$ be the ch.f. of $m, p$, and $m * p$, respectively, where $m * p$ is any convolution of $m$ and $p$. Then

$$
\begin{aligned}
h(t) & =\int_{R} e^{i t z} d m * p(z) \\
& =\int_{R} e^{i t(x+y)} d m * p(x+y)=\int_{R \times R} e^{i t(x+y)} d s(x, y) \\
& =\int_{R \times R} e^{i t x} e^{i t y} d m \times p(x, y)=\int_{R}\left(\int_{R} e^{i t x} d m(x)\right) e^{i t y} d p(y) \\
& =\int_{R} e^{i t x} d m(x) \cdot \int_{R} e^{i t y} d p(y)=f(t) \cdot g(t) .
\end{aligned}
$$

The fifth equality uses Appling's form of Fubini's Theorem. The fourth equality depends on the fact that $e^{i t(x+y)}$ is the product of independent bounded functions $e^{i t x}$ and $e^{i t y}$.

Because of the nonuniqueness of convolutions, it helps to strengthen the requirements for infinitely divisible laws by using ultrafilter limits. So that the usual laws still qualify as infinitely divisible, the Boolean algebra $\mathscr{B}$ will henceforth be the Borel sets. The usual definition, as in Chung [4, Chapter 7], simply requires, $\forall n \in N$, a countably additive infinitely divisible law to be an $n$-fold convolution of countably additive probability measures. A f.a.p.m. $m$ is infinitely divisible iff there are $U$, an ultrafilter on $I \neq \varnothing$, and countably additive probability measures $m_{n, i}$ so that $m_{n}=U-\lim \left(m_{n, i}: i \in I\right)$ and $m=U-\lim \left(m_{n, i}^{* n}: i \in I\right)$ for each $n \in N$. A ch.f. is infinitely divisible iff it is the ch.f. of an infinitely divisible law. All the usual countably additive laws are still infinitely divisible. Simply let $I=\{0\}$ and $m_{n, 0}=$ $m_{n}$, where $m_{n}^{* n}=m$ in the usual definition.

THEOREM 2. The infinitely divisible laws and their ch.f. are closed under ultrafilter limits.

Proof. Suppose for $n \in N, i \in I$ and $j \in J$ that $m_{j, i, n}$ are countably additive probabilities and $U_{j}$ is an ultrafilter on $I$. Let $m_{j}$ be infinitely divisible with $m_{j, n}=U_{j}-\lim \left(m_{j, i, n}: i \in I\right)$ and $m_{j}=U_{j}-\lim \left(m_{j, i, n}^{* n}: i \in I\right)$. Let $f_{j}$ be the ch.f. of $m_{j}$ 
and $V$ be an ultrafilter on $J$. The f.a.p.m.

$$
p=V-\lim \left(m_{j}: j \in J\right)=V-\lim \left(U_{j}-\lim \left(m_{j, i, n}^{* n}: i \in I\right): j \in J\right)
$$

has the ch.f. $V-\lim \left(f_{j}: j \in J\right)$ by the lemma. To show that $p$ is infinitely divisible, it seems natural to use $p_{n}=V-\lim \left(U_{j}-\lim \left(m_{j, i, n}: i \in I\right): j \in J\right)$. However, the definition of an infinitely divisible law only allows the use of one ultrafilter. For $A \subset J \times I$, define $A \in W$ iff $\left\{j: A_{j} \in U_{j}\right\} \in V$, where $A_{j}=\{i \in I:(j, i) \in A\}$.W is then an ultrafilter on $J \times I$,

$$
p=W-\lim \left(m_{j, i, n}^{* n}:(j, i) \in J \times I\right)
$$

and

$$
p_{n}=W-\lim \left(m_{j, i, n}:(j, i) \in J \times I\right) .
$$

Thus $p$ is infinitely divisible.

It is an open question whether the countably additive infinitely divisible laws are dense among all infinitely divisible laws under ultrafilter limits.

\section{BIBLIOGRAPHY}

1. W. D. L. Appling, A Fubini-type theorem for finitely additive measure spaces, Ann. Scuola Norm. Sup. Pisa Cl. Sci. (4) 1 (1974), 155-166.

2. J. L. Bell and A. B. Slomson, Models and ultraproducts: An introduction, North-Holland, Amsterdam, 1969.

3. C. C. Chang and H. J. Keisler, Continuous model theory, Princeton Univ. Press, Princeton, N.J., 1966.

4. K. L. Chung, A course in probability theory, Academic Press, New York, 1974.

5. A. McK. Shorb, Completely additive measure and integration, Proc. Amer. Math. Soc. 53 (1975), 453-459.

6. T. Q. Sibley, The theory of finitely additive probability using non-standard analysis, Ph.D. dissertation, Boston University, 1980.

Department of Mathematics, Cuttington University College, P.O.B. 277, Monrovia, Liberia 